\title{
A efetividade da terapia de liberação posicional (TLP) em pacientes com cervicalgia
}

\author{
The effectiveness of posicional release therapy (PRT) in \\ cervicalgia patients
}

\begin{abstract}
Myria Karina Monteiro de Sobral ${ }^{[a]}$, Priscila Gregório da Silva ${ }^{[b]}$, Ricardo Alexandre Guerra Vieira ${ }^{[\mathrm{c}]}$, Gisela Rocha de Siqueira ${ }^{[\mathrm{d}]}$
\end{abstract}

[a] Fisioterapeuta graduada na Faculdade Associação Caruaruense de Ensino Superior (ASCES), Caruaru, PE - Brasil, e-mail: myria_fisioterapia@yahoo.com.br

${ }^{[b]}$ Fisioterapeuta graduada na Faculdade ASCES (Associação Caruaruense de Ensino Superior), Caruaru - PE, e-mail: priscilagregorio@yahoo.com.br

[c] Mestre em Hebiatria pela Universidade de Pernambuco (UPE), Especialista em Pedagogia dos Esportes e Treinamento Esportivo, educador físico e professor do curso de Educação Física da Faculdade ASCES (Associação Caruaruense de Ensino Superior), Caruaru, PE - Brasil, e-mail: rhandguerra@hotmail.com

[d] Fisioterapeuta, professora adjunta do Departamento de Fisioterapia da Universidade Federal de Pernambuco (UFPE), Doutora em Saúde da Criança e do Adolescente e Mestre em Saúde Coletiva pela UFPE, Caruaru, PE - Brasil, e-mail: giselarsiqueira@gmail.com.br

\section{Resumo}

Introdução: A cervicalgia de origem mecânica é uma disfunção musculoesquelética que acomete número considerável de indivíduos, trazendo prejuízos nas suas atividades de vida diária. Uma das ferramentas da Fisioterapia para o tratamento desta disfunção é a Terapia de Liberação Posicional (TLP), método de tratamento indireto que utiliza pontos sensíveis e uma posição de conforto para solucionar a disfunção associada. Objetivo: Este estudo teve como objetivo comparar o nível de dor, amplitude de movimento e força muscular antes e após a aplicação da TLP. Metodologia: Foi realizado um estudo analítico, intervencional e randomizado, composto por dois grupos de dez indivíduos cada, um intervencional e um controle, formados por pacientes com diagnóstico de cervicalgia que estiveram em atendimento na clínica-escola da ASCES. Resultados: Nos resultados observou-se que houve melhora estatisticamente significativa no grupo da intervenção terapêutica em relação ao nível de dor, amplitude de movimento e força muscular após a aplicação da TLP, apresentando um $\mathrm{p}<0,05$. Conclusão: Diante dos resultados obtidos evidencia-se que a TLP é uma técnica eficaz 
e benéfica, podendo ser aliada aos tratamentos já existentes na disfunção músculo esquelética em pacientes com cervicalgia.

Palavras-chave: Cervicalgia mecânica. Disfunção musculoesquelética. Terapia de liberação posicional.

\begin{abstract}
Introduction: Cervicalgia of mechanical origin is a musculoskeletal dysfunction that affects a lot of people in their daily lives. One of Physiotherapy tools to deal with it is Positional Release Therapy (PRT), an indirect methodology in which tender points and a comfortable position to cure the associated dysfunction are used. Objective: This study's objective was to compare the level of pain, the amplitude of movement and the muscular strength before and after performing PRT. Methods: It was examined following an analytic, interventional and randomized study where two groups, the interventional and the control, were composed of patients diagnosed with cervicalgia under the care of ASCES clinic-school. Results: The results showed a statistically significant improvement in the therapeutic intervention group where examined variables were 100\% accurate. Conclusion: Based upon our results, PRT has proven to be an efficient and beneficial technique that can be used in already existing treatments of cervicalgia patients with musculoskeletal dysfunction.
\end{abstract}

Keywords: Mechanical cervicalgia. Musculoskeletal dysfunction. Posicional release therapy.

\title{
Introdução
}

A cervicalgia acomete um número considerável de indivíduos, com uma média de $12 \%$ a 34\% de uma população adulta em alguma fase da vida, com maior incidência no sexo feminino, trazendo prejuízos nas suas atividades de vida diária (1-4).

Esta patologia raramente se inicia de maneira súbita, em geral pode estar relacionada com movimentos bruscos, longa permanência em posição forçada, esforço ou trauma. Também pode ser definida como uma dor localizada na parte posterior do pescoço e superior das escápulas ou zona dorsal alta, que não se acompanha de sinais característicos de radiculopatia (5-7).

A Terapia de Liberação Posicional (TLP) vem avançando e se desenvolvendo continuamente a partir das contribuições de muitos médicos e pesquisadores. É um instrumento da fisioterapia que visa a uma harmonização no sistema musculoesquelético, cujos princípios englobam técnicas que aproximam origem e inserção muscular passivamente $(8,9)$.

Sua característica principal é o posicionamento específico dos segmentos corporais com a finalidade de aliviar a dor de pontos sensíveis, os quais podem surgir em qualquer tecido somático, como músculos, fáscias, ligamentos, tendões, cápsula articular, sincondroses, suturas cranianas, periósteo e osso. Estes pontos podem ser identificados à palpação sob a forma de nódulos pequenos (de 0,25 a 1,0 $\mathrm{cm}$ ), geralmente localizados nos tecidos subcutâneo, muscular ou fascial, provocando irritação tecidual, tensão muscular e dor. Assim, a finalidade da TLP é beneficiar o paciente no alívio das dores e espasmos musculares (9).

Há hipóteses de que a técnica atua favorecendo o equilíbrio do tônus, pois parece afetar a atividade proprioceptiva inapropriada; a normalização da tensão fascial, ocorrendo uma ação relaxante do tecido; diminuição da hipomobilidade articular, em virtude do relaxamento dos músculos e tecidos fasciais afetados; melhoria da circulação e redução do edema, graças ao relaxamento das estruturas musculoesqueléticas; redução da dor, em resposta ao alívio do espasmo; e aumento da força, uma vez que a técnica restaura o tônus e a função normal dos músculos envolvidos (9).

Apesar de a literatura apontar inúmeros benefícios da TLP no alívio da sintomatologia cervical, existem poucos estudos abordando esta temática. Diante da problemática apresentada, o seguinte trabalho 
de pesquisa foi desenvolvido, com o objetivo de comparar o nível de dor, amplitude de movimento e força muscular em pacientes com diagnóstico de cervicalgia mecânica antes e após a aplicação da TLP.

\section{Metodologia}

Trata-se de um estudo analítico, intervencional e randomizado desenvolvido com pacientes do sexo feminino, na faixa etária de 24 a 40 anos, com diagnóstico de cervicalgia mecânica, que estiveram em atendimento na clínica escola da ASCES no período de 6 de agosto a 26 de setembro de 2008.

A cervicalgia mecânica foi definida como dor referida na cervical associada à tensão da musculatura adjacente e sensibilidade aumentada nos pontos sensíveis identificados à palpação, localizados na parte posterior do pescoço e superior das escápulas ou zona dorsal alta, e ausência de sinais de radiculopatia ou qualquer patologia neurológica e degenerativa (6).

Foram excluídos de estudo todos os pacientes com cervicalgia mecânica que estivessem em tratamento fisioterapêutico ou em uso de medicamentos anti-inflamatórios, analgésicos ou miorrelaxantes no período do estudo ou por no mínimo seis meses anteriores, indivíduos que apresentassem neuralgia cervicobraquial ou cervicobraquialgia, cervicalgia secundária à osteoartrose, espodiliteanquilosante, espodilolistese, hérnia de disco, tumores ou qualquer outra condição patológica que desencadeasse uma cervicalgia ou que impedisse a realização da terapia de liberação posicional.

Foram selecionados 20 pacientes para participarem do estudo, que por meio de sorteio foram alocados em dois grupos, um experimental e um grupo controle, que apresentaram o mesmo número de indivíduos.

Todos os pacientes foram avaliados inicialmente a partir de um questionário elaborado pelos pesquisadores intitulado "Avaliação dos distúrbios cervicais". Este questionário apresenta as seguintes variáveis: presença de dor cervical nos últimos meses, características sociodemográficas (idade, escolaridade, posse de bens, renda), perfil profissional (profissão, situação previdenciária), características da cervicalgia, duração do episódio (horas, um dia, um dia a uma semana, uma semana a um mês, $>1$ mês), e conduta terapêutica (consulta médica, automedicação (medicação obtida sem receita ou aconselhamento médico), tratamento fisioterapêutico ou não convencional (medicinas alternativas - acupuntura, ervanária), autocuidado (massagem, correção postural), outros.

Para a avaliação da intensidade da dor relatada pelos pacientes foi utilizada a Escala Visual Numérica $(\mathrm{EVN})$, descrita por Sousa e Silva (10), que consiste numa escala graduada de zero (0) a dez (10), na qual zero significa ausência de dor e dez, a pior dor imaginável. Os participantes do estudo foram instruídos a marcar o nível de dor sentido nos últimos sete dias.

Os sujeitos também foram submetidos a uma avaliação fisioterapêutica que incluiu anamnese, inspeção na postura em pé (frontal, dorsal e lateral direito e esquerdo), palpação dos pontos sensíveis dos músculos cervicais superficiais, laterais e profundos (trapézio superior, esplênios, escalenos, esternocleidomastoideo, nucais, levantador da escápula e romboides), goniometria da coluna cervical e teste de força muscular. Para esta avaliação os participantes foram orientados a estar com o tórax e região cervical desnuda, utilizando apenas uma faixa elástica para cobrir os seios (11).

Para a palpação dos pontos sensíveis, o paciente foi posicionado em decúbito dorsal ou ventral numa superfície rígida, com os membros inferiores fletidos e os pés apoiados na mesa de exame. Foi realizada a palpação bilateral desses músculos e o indivíduo foi instruído a não realizar nenhuma contração da musculatura cervical.

Para a realização da goniometria, os participantes da pesquisa foram posicionados sentados numa cadeira e o instrumento a ser utilizado foi um goniômetro da marca Trident ${ }^{\circledR}$.

Já para o teste de força do paciente, foram avaliados os seguintes grupamentos musculares: flexores, extensores, rotadores e inclinadores cervicais (12). A força foi classificada em grau 5 (normal), grau 4 (bom), grau 3 (regular), grau 2 (precário), grau 1 (atividade traço), grau 0 (nenhuma atividade).

Após a realização de todas as avaliações, o grupo experimental foi submetido ao tratamento da cervicalgia mecânica com dez sessões de Terapia de Liberação Posicional, realizadas três vezes por semana. 
O primeiro procedimento da técnica foi a identificação de pontos sensíveis (PS) na musculatura cervical, por meio de uma digitopressão suave, e a classificação destes pontos em ordem decrescente de intensidade dolorosa. Após a localização dos OS, a técnica era executada inicialmente no ponto de maior sensibilidade e, logo após, nos pontos subsequentes.

Para a liberação do PS de um determinado músculo, mantinha-se a pressão sobre o ponto e em seguida a cervical do paciente era passivamente e lentamente colocada numa posição de conforto, a partir da aproximação da origem e inserção do referido músculo. O posicionamento e a digitopressão era mantida por intervalo de 90 segundos e após este tempo liberava-se a digitopressão e reposicionava-se a cabeça do participante passivamente e lentamente até a posição neutra. Na mesma sessão, este procedimento era realizado em todos os PS encontrados na palpação e em cada ponto repetia-se a técnica até se obter uma resposta mínima de alívio dos sintomas de 70\% ao fim da sessão, quando comparada ao início desta (9).

No grupo controle não foram realizados procedimentos fisioterapêuticos e nem procedimentos relativos à estabilização segmentar durante o período de estudo. Após o término das dez sessões, foi realizada uma nova reavaliação fisioterapêutica nos dois grupos, para uma análise das variáveis em estudo.

As variáveis categóricas foram apresentadas como frequências e percentuais e as variáveis contínuas como média e desvio padrão (técnicas de estatística descritivas). Verificou-se a distribuição da normalidade para as variáveis quantitativas por meio do teste Komogorov-Smirnov Z. Utilizou-se o teste t-Student pareado para analisar as diferenças do mesmo grupo; o teste t-Student não pareado foi considerado na análise para comparações entre o grupo controle e de intervenção (técnicas de estatística inferencial). A verificação da hipótese de igualdade de variâncias foi realizada por meio do teste F de Levene. Considerou-se o nível de significância $\alpha=0,05$. Os dados foram digitados na planilha Excel e o software utilizado para a obtenção dos cálculos estatísticos foi o SPSS ${ }^{\circledR}$ (Statistical Package for the Social Scienc) na versão 13.0.

Este estudo foi aprovado pelo comitê de ética em pesquisa da Associação Caruaruense de Ensino Superior (ASCES). Após o término da coleta de dados e finalização da pesquisa, os pacientes do grupo controle foram submetidos a dez sessões de TLP, de acordo com os mesmos princípios aplicados ao grupo experimental.

\section{Resultados}

Todos pacientes que foram selecionados para fazer parte dos grupos intervencional e controle completaram o período determinado para o tratamento, não havendo perda de segmento do estudo. Os participantes da pesquisa eram todos do sexo feminino $(n=20 / 100 \%)$, não faziam uso de medicações e apresentavam o nível de dor e tensão muscular aumentado, assim como mobilidade cervical e nível de força reduzida. Sessenta e cinco por cento $(\mathrm{n}=13)$ das pacientes eram casadas e 35\% (n $=7$ ) eram solteiras. Observando a escolaridade, verificamos que $15 \%(\mathrm{n}=3)$ possuíam ensino fundamental completo, $35 \%(\mathrm{n}=7)$ ensino médio e $50 \%(n=10)$ ensino superior.

Quarenta por cento $(n=8)$ das pacientes avaliadas trabalhavam no setor administrativo, $25 \%(n=5)$ na docência, $15 \%(\mathrm{n}=3)$ no lar e $20 \%(\mathrm{n}=4)$ eram costureiras. A idade média no grupo experimental foi de 33,2 \pm 5,0 anos, apresentando como idade mínima de 25 anos e máxima de 40. Para o grupo controle, a média consistiu em 31,50 \pm 5,4 anos, com mínima de 24 e máxima de 39 anos de idade.

Foram encontradas diferenças significativas ao término do estudo nos dois grupos da análise em relação à dor, amplitude de movimento e força muscular. Em relação à análise da dor, pela escala visual analógica, 100\% das pacientes do grupo intervencional relataram melhora ao fim do tratamento. Quanto ao grupo controle, $20 \%$ dos pacientes referiram diminuição da dor, 40\% permaneceram e 40\% pioraram. Na avaliação da força, pelo teste de força manual, no grupo intervencional 100\% das pacientes apresentaram aumento do nível de força; já no grupo controle, todas as pacientes permaneceram no mesmo nível.

Em relação à ADM, realizada por meio da goniometria, no grupo intervencional 100\% das pacientes aumentaram os graus em todos os planos de movimento; já no grupo controle $20 \%$ diminuíram sua ADM, $40 \%$ permaneceram e $40 \%$ aumentaram sua ADM, entretanto com pouca significância estatisticamente, com exceção do movimento de inclinação lateral à direita. 
Verificou-se na Tabela 1 que, para as variáveis da amplitude do movimento e nível de dor, tanto o grupo controle quanto o de intervenção não apresentaram diferenças significativas ao início do estudo, exceto para o movimento de inclinação lateral direita inicial ( $\mathrm{p}<0,014)$. Observou-se que para todos os movimentos finais houve diferença significativa entre os grupos experimental e controle, assim como para o nível de dor $(\mathrm{p}<0,0001)$.

Tabela 1 - Distribuição da média e desvio-padrão da amplitude de movimento e nível de dor segundo grupo de intervenção e controle. Caruaru, PE - Brasil, 2008

\begin{tabular}{|c|c|c|c|c|}
\hline \multirow{3}{*}{ Variáveis } & \multicolumn{2}{|c|}{ Grupo } & \multirow{3}{*}{$\mathrm{T}$} & \multirow{3}{*}{ Valor de 1} \\
\hline & Intervenção $(n=10)$ & Controle $(n=10)$ & & \\
\hline & média $\pm d p$ & média $\pm d p$ & & \\
\hline Flexão inicial & $33,90 \pm 4,48$ & $34,60 \pm 5,35$ & $0,317(1)$ & 0,755 \\
\hline Flexão final & $56,505 \pm 3,62$ & $35,30 \pm 6,09$ & $9,454(1)$ & $0,0001 *$ \\
\hline Extensão inicial & $27,10 \pm 4,58$ & $27,00 \pm 6,20$ & $0,041(1)$ & 0,968 \\
\hline Extensão final & $44,60 \pm 2,95$ & $27,30 \pm 4,88$ & $9,596(1)$ & $0,0001 *$ \\
\hline Rotação Direita inicial & $26,90 \pm 5,38$ & $30,40 \pm 3,53$ & $1,71(1)$ & 0,103 \\
\hline Rotação direita final & $49,40 \pm 49,40$ & $30,90 \pm 3,35$ & $13,651(1)$ & $0,0001 *$ \\
\hline Rotação esquerda inicial & $26,00 \pm 7,19$ & $26,70 \pm 5,81$ & $0,239(1)$ & 0,814 \\
\hline Rotação esquerda final & $49,80 \pm 1,98$ & $27,10 \pm 6,29$ & $10,870(2)$ & $0,0001 *$ \\
\hline Inclinação lateral direita inicial & $18,90 \pm 3,87$ & $22,90 \pm 2,60$ & $2,71(1)$ & $0,014^{*}$ \\
\hline Inclinação lateral direita final & $33,80 \pm 2,61$ & $24,80 \pm 2,57$ & $7,756(1)$ & $0,0001 *$ \\
\hline Inclinação Lateral esquerda inicial & $21,50 \pm 3,80$ & $22,60 \pm 2,87$ & $0,729(1)$ & 0,475 \\
\hline Inclinação lateral esquerda final & $34,30 \pm 2,58$ & $24,30 \pm 3,23$ & $7,640(1)$ & $0,0001 *$ \\
\hline Nível de dor inicial & $9,30 \pm 0,823$ & $9,20 \pm 0,79$ & $0,277(1)$ & 0,785 \\
\hline Nível de dor final* & $1,20 \pm 0,92$ & $9,40 \pm 0,51$ & $24,600(1)$ & $0,0001 *$ \\
\hline
\end{tabular}

(*) Diferença significante a 5\%.

(1) Por meio do teste t-Student com variâncias iguais.

(2) Por meio do teste t-Student com variâncias desiguais.

Verificando-se as diferenças das médias da amplitude de movimento dentro de cada grupo, observou-se que as diferenças estatisticamente significativas residiram no grupo da intervenção terapêutica, destacando-se a flexão e a inclinação lateral com as maiores médias ( $\mathrm{p}<0,001)$. No entanto, o grupo controle apresentou a maior diferença no movimento de inclinação lateral direita $(p=0,038)$, como se observa na Tabela 2. 
Tabela 2 - Distribuição das diferenças pareadas da média e desvio-padrão da amplitude de movimento e nível de dor segundo grupo de intervenção e controle. Caruaru, PE - Brasil, 2008

\begin{tabular}{|c|c|c|c|c|c|c|}
\hline \multirow[b]{2}{*}{ Variáveis } & \multicolumn{6}{|c|}{ Diferença pareada Grupo } \\
\hline & $\begin{array}{c}\text { Intervenção } \\
(\mathrm{n}=10) \\
\text { Media } \pm \mathrm{dp}\end{array}$ & $\mathbf{t}$ & $\mathrm{p}^{(1)}$ & $\begin{array}{c}\text { Controle } \\
(n=10) \\
\text { Media } \pm d p\end{array}$ & $\mathbf{t}$ & $\mathrm{p}^{(1)}$ \\
\hline Flexão inicial-final & $22,60 \pm 2,01$ & 35,537 & $0,0001 *$ & $0,70 \pm 2,49$ & 0,887 & 0,398 \\
\hline Extensão inicial - final & $17,50 \pm 3,83$ & 14,423 & $0,0001 *$ & $0,30 \pm 2,94$ & 0,322 & 0,755 \\
\hline Rotação direita inicial - final & $22,50 \pm 6,96$ & 10,217 & $0,0001 *$ & $0,50 \pm 2,46$ & 0,643 & 0,537 \\
\hline Rotação esquerda inicial - final & $23,80 \pm 6,47$ & 11,619 & $0,0001 *$ & $0,40 \pm 2,17$ & 0,583 & 0,574 \\
\hline Inclinação lateral direita inicial - final & $14,90 \pm 1,37$ & 34,385 & $0,0001 *$ & $1,90 \pm 2,47$ & 2,433 & $0,038^{*}$ \\
\hline Inclinação lateral esquerda inicial - final & $12,80 \pm 1,75$ & 23,114 & $0,0001 *$ & $1,70 \pm 2,40$ & 2,234 & 0,052 \\
\hline Nível de dor inicial final & $8,1 \pm 0,31$ & 81,0 & $0,0001 *$ & $0,20 \pm 0,78$ & 0,80 & 0,443 \\
\hline
\end{tabular}

(*) Diferença significante a $5 \%$.

(1) Por meio do teste t-Student para amostras pareadas.

\section{Discussão}

A Fisioterapia dispõe de várias técnicas manuais que podem proporcionar o alívio da dor, restabelecendo as propriedades estruturais e funcionais do tecido, e que também podem afetar o fluxo de fluidos, reduzindo as obstruções estruturais no interior do tecido. A TLP é uma dessas técnicas que têm mostrado resultados bastante satisfatórios e imediatos no alívio da dor em pacientes com cervicalgia (13-15).

De acordo com os resultados do presente estudo, verificou-se redução significativa na dor e na tensão muscular e aumento na ADM e força muscular dos pacientes com cervicalgia tratados com a TLP.

Na cervicalgia mecânica, a transmissão da dor no paciente começa com a liberação de mediadores químicos pró-inflamatórios e vasoconstrictores, que estimulam os nociceptores, órgãos sensoriais localizados na extremidade de pequenos neurônios não mielinizados ou levemente mielinizados, sensíveis ao mínimo estímulo. Além disso, a dor cervical também pode ser oriunda da tensão muscular que aparece em decorrência do acúmulo de ácido lático, em virtude do esforço repetitivo, posturas inadequadas ou contração estática mantida, o que pode levar à ativação dos pontos sensíveis e desencadear a formação de nódulos de tensão e provocar atividade reflexa de dor mesmo sem a palpação $(9,16,17,18)$.

De acordo com D'Ambrógio (5), a posição de conforto mantida pela TLP, que consiste na aproximação de origem e inserção muscular, promove a diminuição da ativação do fuso neuromuscular, redução da tensão sobre o sistema miofascial e, consequentemente, o relaxamento muscular. Isto se dá a partir da liberação das ligações cruzadas colagenosas e da ruptura das ligações eletroquímicas, reduzindo a estimulação dos receptores da dor.

Por outro lado, a TLP envolve a digitopressão nas áreas localizadas como pontos deflagradores, usando um dedo polegar ou indicador, ou ainda um dedo médio, reforçado por outros dedos. Esta pressão manual pode também induzir a analgesia por meio de um processo neurológico chamado bloqueio sensorial. No bloqueio sensorial, o processamento e a percepção de uma modalidade sensorial podem ser reduzidos por um estímulo concomitante sobre o outro (13). 
Ao considerar a ADM, verificou-se no grupo intervencional um ganho estatisticamente significante em todos os movimentos, o que não ocorreu no grupo controle. Estudos demonstram que após o uso da TLP os músculos e os tecidos fasciais relaxam, o que parece reduzir a tensão periarticular, permitindo que a articulação se mova mais livremente, restaurando o movimento biomecânico adequado da articulação $(9,19)$.

O ganho na amplitude de movimento referida é conseguido pela liberação miofascial. Ao aproximar o tecido conjuntivo, que é composto por colágeno e elastina, há uma diminuição da produção de colágeno. Logo, a quantidade de fibras de elastina será mais concentrada e armazenada em energia potencial e se transformará em energia térmica, que irá aquecer e liquefazer a substância fundamental amorfa, transformandose depois em energia cinética, aumentando a amplitude de movimento do tecido conjuntivo, que retorna à posição inicial (20-22).

Em relação ao nível de força muscular, todos os pacientes submetidos à TLP aumentaram o grau de força. Esse fato acontece graças à normalização do equilíbrio proprioceptivo e neural do tecido muscular e da remoção causada pela dor (23-26). A terapia de liberação posicional pode ajudar a restaurar o tônus e a função normal dos músculos envolvidos, portanto, esta técnica pode otimizar a eficiência biomecânica do músculo (9).

Para Domenico (20), isso acontece porque a fáscia ajuda a manter a força muscular; logo, a liberação miofascial reduz a constrição e a dor sem comprometer a resistência muscular.

Foi constatado que após a aplicação da técnica de liberação no grupo intervencional, houve melhora quanto aos aspectos clínicos analisados no estudo, ou seja, dor, amplitude de movimento e força muscular.

Quanto ao grupo controle, que permaneceu durante todo o período de estudo sem tratamento, apresentou aumento nos níveis de dor pela EVA. Isto pode se dar em virtude da manutenção da tensão muscular, diminuição da mobilidade e encurtamento dos músculos cervicais, o que em longo prazo pode promover a redução do número de sarcômeros (40\% em poucos dias), edema excessivo e estase nos espaços dos tecidos, aumentando a dor e, consequentemente, diminuindo a amplitude de movimento e força muscular (13, 27-30).

\section{Considerações finais}

De acordo com os dados apresentados, foram observados resultados estatisticamente significantes após a aplicação da Técnica de Liberação Posicional nos pacientes estudados no grupo da intervenção terapêutica, em relação à diminuição do nível de dor, aumento da amplitude de movimento e da força muscular. Destaca-se, portanto, a importância da utilização da TLP como um procedimento aliado aos tratamentos de fisioterapia convencional nas disfunções musculoesqueléticas em pacientes com cervicalgia. Isto sugere a necessidade de a comunidade científica investir em estudos sobre esta técnica e sua introdução na rotina dos profissionais fisioterapeutas em busca de soluções para o tratamento de distúrbios osteomusculares.

\section{Referências}

1. Cote P, Cassidy J, Carroll L. The factors associated with neck pain and its related disability in the Saskatchewan population. Spine. 2000;25(9):1109-17.

2. Palmer KT, Walker-Bone K, Griffin MJ, Syddall H, Pannett B, Coggon D, et al. Prevalence and occupational associations of neck pain in the British population. Scand J Work Environ Health. 2001;27(1):49-56.

3. Teixeira JM, Barros Filho T, Lin TY, Hamani C, Teixeira WGJ. Cervicalgias. Rev Med. 2001;80(Pt 2):307-16.

4. Salomão Junior JC, Zardo EA, Serdeira A, Paglioli E. Cervicobraquialgia. Acta Médica. 2004;25:26-37.

5. Kraychete DC, Sakata RK, Tanajura D, Guimarães AC, Angelim M. Perfil clínico de pacientes com dor crônica do ambulatório de dor do Hospital Universitário Professor Edgard Santos - UFBA. Rev Baiana Saúde Pública. 2003;27:185-95. 
6. Gabriel MRS, Petit JD, Carril MLS. Fisioterapia em traumatologia, ortopedia e reumatologia. Rio de Janeiro: Revinter; 2001.

7. Herbert S, Xavier R, Pardini Jr. AG, Barros Filho TEP. Ortopedia e traumatologia: princípios e prática. Porto Alegre: Artmed; 2003.

8. Castro FM, Gomes RCV, Salomão JR, Abdon APV. A efetividade da Terapia de Liberação Posicional (TLP) em pacientes portadores de disfunção temporomandibular. Revista de Odontologia da Universidade de São Paulo. 2006;18(1):67-74.

9. D’Ambrogio KJ, Roth GB. Terapia de liberação posicional: avaliação e tratamento da disfunção músculo esquelética. São Paulo: Manole; 2001.

10. Souza FAEF, Silva JA. A métrica da dor (dorimetria): problemas teóricos e metodológicos. Rev Dor: Pesquisa, Clínica e Terapêutica. 2005;6(1):469-513.

11. Dutton M. Fisioterapia ortopédica: exame, avaliação e intervenção. Porto Alegre: Artmed; 2006.

12. Hislop HJ, Montgomery J. Provas de função muscular: técnicas de exame manual. Rio de Janeiro: Guanabara Koogan; 1996.

13. Lenderman E. Fundamentos da terapia manual: fisiologia, neurologia e psicologia. São Paulo: Manole; 2001.

14. Cleland JA, Childs JD, McRae M, Palmer JA, Stowell T. Immediate effects of thoracic manipulation in patients with neck pain: a randomized clinical trial. Man Ther. 2005;10(2):127-35.

15. Di Fabio RP. Manipulation of the cervical spine: risks and benefits. Phys Ther. 1999;79(1):50-65.

16. Wright A, Mayer T, Gatchel R. Outcomes of disabling cervical spine disorders in compensation injuries: a prospective comparison to tertiary rehabilitation response for chronic lumbar disorders. Spine. 1999;24(2):178-83.

17. Cote P, Cassidy JD, Carroll LJ, Kristman V. The annual incidence and course of neck pain in the general population: a population-based cohort study. Pain. 2004;112(3):267-73.

18. David C, Lloyd J. Rheumatological physiotherapy. London: Premier, 2001.

19. Yeng LT, Teixeira MJ, Kaziyama HHS. Síndrome dolorosa miofascial. Rev Med. 2001;80(Pt 1):94-110.

20. Domenico G, Wood EC. Técnicas de massagem de Beard. São Paulo: Manole; 1998.

21. Gross AR, Hoving JL, Haines TA, Goldsmith CH, Kay T, Aker P, et al. Manipulation and mobilisation for mechanical neck disorders. Cochrane Database Syst Rev. 2004;(1):CD004249.

22. Haraldsson BG, Gross AR, Myers CD, Ezzo JM, Morien A, Goldsmith C, et al. Massage for mechanical neck disorders. Cochrane Database Syst Rev. 2006;3:CD004871.

23. Svensson P, Wang K, Sessle BJ, Arendt-Nielse L. Associations between pain and neuromuscular activity in the human jaw and neck muscles. Pain. 2004;109(3):225-32.

24. Pool JJ, Ostelo RW, Koke AJ, Bouter LM, de Vet HC. Comparison of the effectiveness of a behavioural graded activity program and manual therapy in patients with sub-acute neck pain: design of a randomized clinical trial. Man Ther. 2006;11(4):297-305.

25. Hoving JL, Koes BW, de Vet HC, van der Windt DA, Assendelft WJ, van Mameren H, et al. Manual therapy, physical therapy, or continued care by a general practitioner for patients with neck pain. A randomized, controlled trial. Ann Intern Med. 2002;136(10):713-22.

26. Vernon H, Humphreys K, Hagino C. Chronic mechanical neck pain in adults treated by manual therapy: a systematic review of change scores in randomized clinical trials. J Manipulative Physiol Ther. 2007;30(3):215-27. 
27. Borghouts JA, Koes BW, Bouter LM. The clinical course and prognostic factors of non-specific neck pain: a systematic review. Pain. 1998;77(1):1-13.

28. Gross AR, Aker PD, Quartly C. Manual therapy in the treatment of neck pain. Rheum Dis Clin North Am. 1996;22(3):579-98.

29. Hoving JL, de Vet HC, Twisk JW, Deville WL, van der Windt D, Koes BW, et al. Prognostic factors for neck pain in general practice. Pain. 2004;110(3):639-45.

30. Hoving JL, de Vet HC, Koes BW, Mameren H, Devillé WL, van der Windt D, et al. Manual therapy, physical therapy, or continued care by the general practitioner for patients with neck pain: long-term results from a pragmatic randomized clinical trial. Clin J Pain. 2006;22(4):370-7.

Recebido: 19/03/2009

Received: 03/19/2009

Aprovado: 29/06/2010

Approved: 06/29/2010 